\title{
Simulation Research of Space-Time Evolution of Emergency Logistics Network Reliability Based on Complex Network Theory
}

\author{
Li Huang, ${ }^{1}$ Wei Wang, ${ }^{2}$ and Minggong Wang ${ }^{2}$ \\ ${ }^{1}$ School of Public Administration, Hohai University, Nanjing, Jiangsu 210098, China \\ ${ }^{2}$ Water Transportation Planning \& Logistics Engineering Institution, College of Harbor, Coastal and Offshore Engineering, \\ Hohai University, Nanjing, Jiangsu 210098, China
}

Correspondence should be addressed to Wei Wang; 13813826667@163.com

Received 21 April 2013; Revised 23 July 2013; Accepted 8 August 2013

Academic Editor: Zhigang Jiang

Copyright (C) $2013 \mathrm{Li}$ Huang et al. This is an open access article distributed under the Creative Commons Attribution License, which permits unrestricted use, distribution, and reproduction in any medium, provided the original work is properly cited.

\begin{abstract}
We propose the conception and evaluation indexes of emergency logistics network connecting reliability to construct evaluation index system of complex network reliability, and describe these indexes quantitatively to evaluate the network connecting reliability. Moreover, the network topological model and the simulation methods of reliability measurement when the network is under attack are present. Finally, we take three classical emergency logistics networks as examples, and through emulation analysis we obtain the connecting reliability changing situation of these three networks under random attack, the changing curve of the ratio of effective demand nodes and emergence supply mileage of emergency logistics network with same network density but different forms, and then evaluate the emergency logistics network connecting reliability. This can provide references for the designing of emergency logistics network with high reliability and analysis means for research in other fields.
\end{abstract}

\section{Introduction}

Emergency logistics refer to special logistic activities with emergence material supply for sudden incidents arising from natural disasters, public health incidents, and so forth as its goals and the optimization of time efficiency and minimization of damages as its objectives and features emergence, uncertainty, weak economy, and unconventionality [1]. Whether emergence materials can be transferred to demand nodes on time upon the arising of emergence incidents will affect results of the whole rescue activities directly. It is of vital importance to design emergency logistics network scientifically for the efficiency of the network which lies on its scale, structure, and layout. As a typical complex network, it may fall as the victim of attacks and damages of different degrees arising from such uncertainties as randomness, diffusivity, and aftermath of the outbreak of emergencies, which may cause local failure or paralysis of the network and affect emergence material supply. It is difficult to work out necessary and universal rules of connecting status in the situation of local failure of the network, so it is a necessity to deepen emergency logistics network reliability research.
The existing huge amount of logistics research mainly focuses on normal logistics activities, but research on abnormal situations of disasters, natural or manmade, is little. Many American researchers studied the logistics supply of America in the Second World War after the end of the war and issued their views on emergency logistics; then the concepts of emergency logistics began to spread in Europe and Japan [2]. Some researchers conducted pilot studies on emergency logistics network optimization. Most of these studies are based on logistics status which is known or can be predicted, and optimization models focus on time, distance, or cost; it is presumed that the road is always in service with utmost transportation capacity, and network structures are fixed in these models; however, little attention is paid to logistics network reliability.

Logistics network reliability reflects the adaptability, antijamming capacity, stability, and recovery capability of emergency logistics network and is of vital importance in ensuring normal function and well performance of the system. Due to the complexity and particularity of emergency logistics network, it is difficult to have quantitative analysis in research, to ascertain problems and optimize the network; at present, 
the research base is very weak and at initial stage, with its definition, methods, models, and theories unshaped [3] and at developing stage. Logistics network is usually constructed with a set of routes and a set of nodes; there are multiple carriers to provide transportation service on each route. Logistics network according to the carrier selection is a typical stochastic-flow network [4] and called a stochastic logistics network (SLN) herein. The network reliability is a performance index of the freight delivery for supply chain management and is defined as the probability that $\mathrm{d}$ units of commodity can be transmitted from a supplier (origin $\mathrm{O}$ ) to a customer (destination D) through a logistics network [5]. Several researchers, such as Aven, Jane, and Lin, have evaluated the network reliability in terms of minimal paths (MPs) or minimal cuts (MCs) [6, 7] without considering unreliable nodes, and Lin has evaluated the network reliability with unreliable nodes in terms of MPs [8] and MCs [9], respectively. Gong et al. construct the operation model and its evaluation index system for emergency logistics system [10].

From the viewpoint of optimization, Levitin and Lisnianski [11] classified the network reliability optimization problems into two categories: minimizing the resources required for providing a specific network reliability level and achieving the maximal network reliability subject to various constraints. Many researchers have discussed the issues involving the network structure, the flow assignment, and the commodity allocation [11-14]. Xu et al. developed a flow assignment strategy with the minimal time elapse and the maximal network reliability, where the carriers on all routes are fixed [15]. Chang et al. formulated the flood emergency logistics problem with uncertainty as two stochastic programming models that allow for the determination of a rescue resource distribution system for urban flood disasters [16]. Lin and Yeh discusse the optimal carrier selection problem based on network reliability criterion [17]. Reliability and spare parts logistics are optimized using heuristic algorithms [18]. Vahdani et al. develop a biobjective model for designing a reliable network of bidirectional facilities in CLSCs under uncertainties [19]. Sheu presents a dynamic relief-demand management model for emergency logistics operations under imperfect information conditions in large-scale natural disasters [20]. Peng et al. model a reliable logistics network design problem with p-robustness constraints and propose a hybrid metaheuristic algorithm to efficiently solve the problem; then substantial improvements in reliability are achievable with minimal increases in cost [21].

Many foreign researchers have studied the supply chain and its measurement methods and got some results. There are mainly two kinds of methods of supply chain reliability measurement, one is experience index measurement, and the other is application reliability engineering index measurement [22]; for example, Hong-Minh et al. studied abrupt logistics by emulation, and Thomas applied reliability engineering in supply chain for the first time and issued the definition of supply chain reliability [23]. Lin developed a MP approach to measure the network reliability for twocommodity supply chain network [24]. Given the fact that emergency logistics can be taken as a kind of special supply chain, the measurement of supply chain reliability is of great value for emergency logistics reliability measurement [25, 26].

With the application of complex network theory, the power failure in North America was examined accurately, which provides a brand new idea for reliability research of local emergency logistics. This essay, based on complex network theories, introduces reliability assessment indexes to assess emergency logistics network reliability and obtains, through emulation attack program development, the changing regularity of emergency logistics network reliability of different types under random attacks. Emulation and analysis methods used in this essay can provide theoretic base and methods for the design and construction of emergency logistics networks and improve the reliability and efficiency of existing emergency logistics networks at a lower cost.

\section{Connecting Reliability of Emergency Logistics Network and Its Assessment Indexes}

The connecting reliability of emergency logistics network refers to the connecting state between supply nodes and demand nodes when suffering from outer interference. Nodes in the network are divided into supply nodes which send emergence materials in response to emergence demand and demand nodes which need these materials according to features of the network. The connecting reliability reflects whether emergence materials can reach demand nodes from supply nodes when such demand rises; obviously, it is closely related to the topological structure of the network except for attack influence. The attention of this essay mainly goes to emergence supply mileage and the ratio of effective demand nodes, two main indexes in the assessment of connecting reliability of the network.

2.1. Emergence Supply Mileage L. Emergence supply mileage, a measurement of timeliness of the whole network fulfilling emergency logistics supply, is defined as the arithmetic mean value of the mileage of all demand nodes in the network, among which the mileage of a demand node refers to the minimum value it needed for obtaining emergence materials:

$$
L=\frac{\sum_{t} \min \left(D(1, t), D(2, t), \ldots, D(s, t), \ldots, D\left(n_{s}, t\right)\right)}{n_{d}}
$$

where $n_{d}$ is all demand node number, $n_{s}$ is all supply node number, and $D(s, t)$ is the mileage from demand node $t(t=$ $\left.1,2, \ldots, n_{d}\right)$ to supply node $s\left(s=1,2, \ldots, n_{s}\right)$.

2.2. Effective Demand Node Ratio P. Effective demand node ratio refers to the ratio of effective demand node number among all demand node number, in which effective demand nodes refer to demand nodes with direct or indirect links to supply nodes, that is, demand nodes which can obtain emergency supply in time:

$$
P=\frac{n_{d}^{\prime}}{n_{d}}
$$


where $n_{d}$ is all demand node number, $n_{d}^{\prime}$ is effective demand node number.

\section{Emulation Methods of Emergency Logistics Network Attack}

3.1. Topological Model of Emergency Logistics Network. Based on the physical structure of actual emergency logistics network, its topological model consists of nodes abstracted from supply nodes and demand nodes in the network, edges abstracted from zones with direct links by real transportation means, and weight of edges which can be seen as transportation mileage or time. In Figure 1, node 1 in the topological structure represents a supply node and the rest represent demand nodes; the figure shows that supply node 1 connects with demand nodes 2,3 , and 5 and but not with demand node 4.

The link between a supply node and a demand node includes direct link (e.g., demand nodes numbers 2 and 3 link up supply node 1 directly) or indirect link (e.g., demand node number 5 links the supply node via demand node number 2). Demand nodes in both the above-mentioned cases are effective nodes. However, those demand nodes with no links, direct or indirect, to supply nodes are not effective demand nodes (e.g., demand node number 4). The weight on edges between nodes represents transport distance or time between nodes.

Now the network can be shown by an adjacent matrix:

$$
D(n)=\left[d_{i j}\right]_{n \times n} .
$$

$d_{i j}$ is the actual distance between node $i$ and node $j$ if there is a direct link between node $i$ and node $j$, and in case of no direct link between node $i$ and node $j$, then $d_{i j}=\infty ; n$ is the total number of nodes $\left(n=n_{s}+n_{d}\right)$.

A topological model of emergency logistics network consisting of supply nodes, demand nodes, and edges is built up. The model keeps topological features of emergency logistics networks, and via the analysis of its connecting reliability the connecting state of the network under attack can be judged.

3.2. Attack Types of Emergency Logistics Network. This essay only studies the situation of nodes in the network under attack. The failure of a node means failure of all zones connecting to it at the same time, and all roads bypassing the node close.

According to the importance of places under attack and attack sequence, attacks can be divided into random attack and target attack.

(1) Random Attack. Attacks happen at all nodes (including supply nodes and demand nodes), for instance, under the situation of natural disasters, incidents, local failures, and so forth.

(2) Target Attack. Target attacks happen in the sequence of nodes with more connections to fewer connections, for instance, in case of terrorist attack and blocking at important nodes.

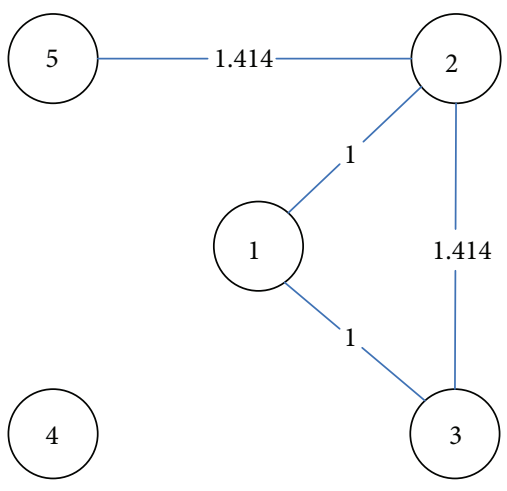

FIGURE 1: Topological model of emergency logistics network.

Given that natural disasters happen randomly, this essay only analyzes the changing regularity of emergency logistics network reliability under random attack.

\subsection{Emulation Analysis of Random Attack}

Random Attack Emulation. Random attack targets a certain node in a network randomly, and edges linking up to it lose efficiency at the same time. The shortest supply mileage from supply nodes to demand nodes is computed according to Dijkstra [27]. Those demand nodes with supply mileage lower than $L_{0}$ (maximal supply mileage) are defined as effective demand nodes in developing emulation programs, and the number and ratio of effective demand nodes in residual network are obtained; emergence supply mileage $L$ of the network is figured up by calculating the arithmetic mean value of supply mileage of all demand nodes. Next, by choosing and attacking one of the nodes in residual network randomly, the effective demand nodes ratio $P$ and emergence supply mileage $L$ of residual network are calculated. These procedures are repeated again and again until all nodes are attacked. The following are steps of emulation computation.

(1) Initialize an adjacent matrix: $D(n)=\left[d_{i j}\right]_{n \times n}$. $d_{i j}$ is the actual distance between node $i$ and node $j$ if there is a direct edge between the two, and in case of no direct link between the two $d_{i j}=L_{0}$.

(2) Generate an integral numeral $r_{1}$ in $[1, n]$; then

$$
D\left(r_{1}, j\right)=D\left(j, r_{1}\right) L_{0} \quad(j=1,2, \ldots, n) .
$$

(3) Programs for counting shortest supply mileage are developed on Visual Basic 6.0 platform according to Dijkstra, and the shortest supply mileage $c[k(m)]$ from supply nodes to all demand nodes is counted out, in which $m$ represents the times taken to count the node of shortest mileage in Dijkstra starting from supply nodes outward step by step, and $k(m)$ is the serial number in the search.

(4) If the supply mileage of a certain demand node $c[k(m)]<L_{0}$, then the node can be marked as an effective demand node, and the number and ratio $P$ of effective demand nodes can be obtained; emergence 


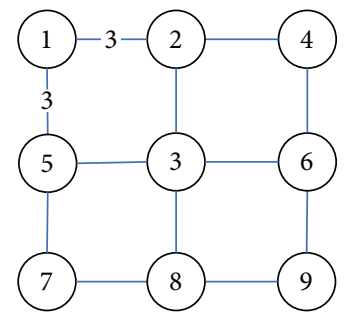

(a) Pane

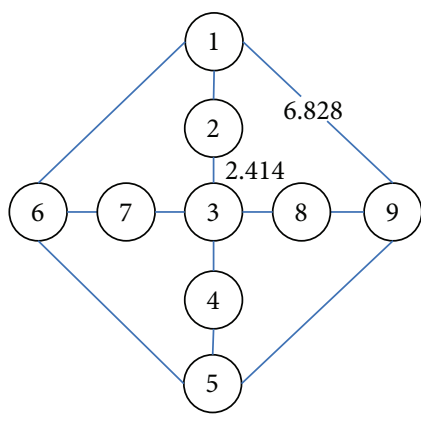

(b) Radial with loop

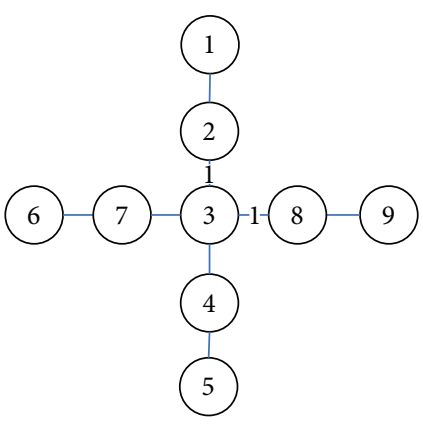

(c) Radial without loop

FIGURE 2: Three forms of heuristic emergency logistics network.

supply mileage $L$ of the network is figured up by calculating the arithmetic mean value of minimum supply mileage $c[k(m)]$ of all demand nodes.

(5) Generate another integral number $r_{2}$ in $[1, n]$ after removal of $r_{1}$ randomly, and $D\left(r_{2}, j\right)=D\left(j, r_{2}\right)=L_{0}$.

(6) Return to step (3) until all integral numbers in $[1, n]$ are taken.

\section{Examples of Heuristic Emulation Analysis}

In order to study effects of emergency logistics network topological structure on its reliability, three classical forms of emergency logistics network are built (shown in Figure 2). All of these emergency logistics networks consist of 9 nodes, one is a supply node, and the rest are demand nodes. The weight on an edge between two nodes represents the mileage of the two nodes; here suppose $L_{0}=120$. Though with different structures (pane, radial with loop, and radial without loop), the three forms of network have same network density, that is, the same ratio of total passage mileage between network nodes to network coverage; here suppose that all the network density is 1 .

The reliability of every form of emergency logistics network is studied by emulation methods proposed above, and emulation results are shown in Tables 1 and 2. Among which letters in network numbering represent network forms, and numerical parts represent the number of supply nodes; for instance, "al" shows the situation of number 1 supply node in a-form (pane) network.

In order to show the change regularities of emergence network reliability of different forms more visually, comparison is made between emulation results of supply nodes with network number 1 in three forms of emergency logistics network a (pane), b (radial with loop) and c (radial without loop). Details are shown in Figures 3 and 4.

The above emulation result shows the following.

(1) Network efficiency changes in line with the selection of different supply nodes in the same network form. Network efficiency stands the highest with supply node number 3 , followed by supply node number 2 , and supply node number 1 the lowest in all the three forms of network from the comparison of emergence

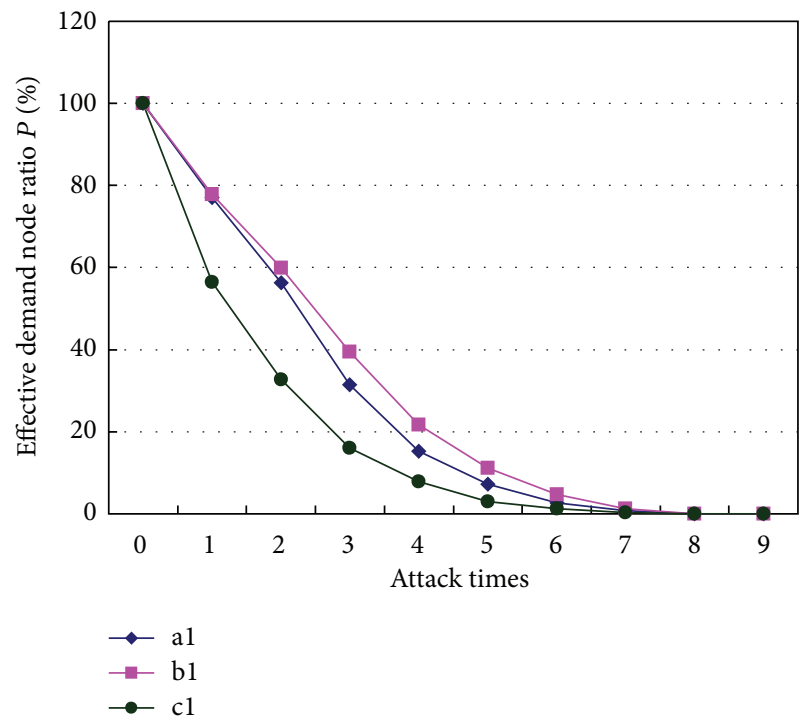

FIgURE 3: Comparison of effective demand node ratio changes of three different forms of network.

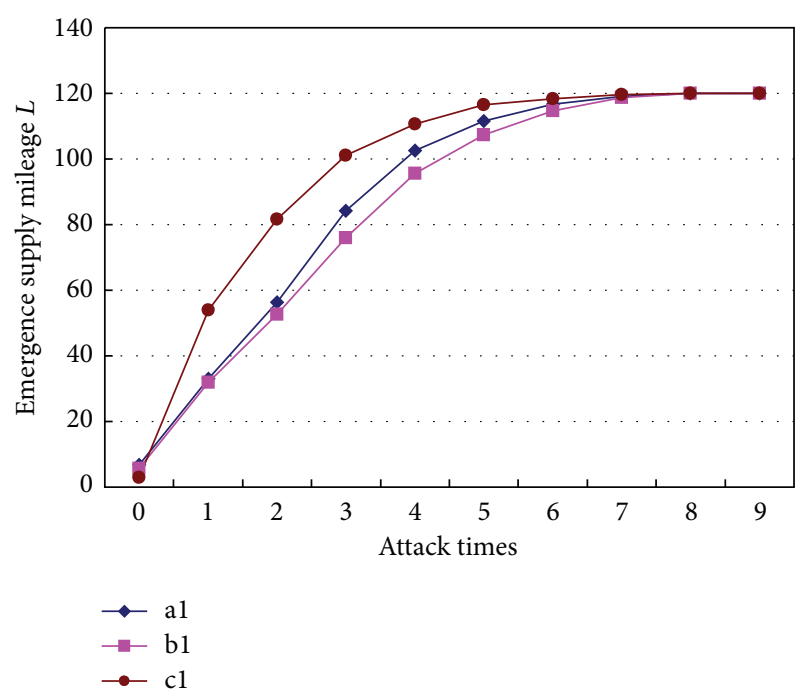

FIGURE 4: Comparison of emergence supply mileage changes of three different forms of network. 
TABLE 1: Changes of effective supply node ratio $P$.

\begin{tabular}{|c|c|c|c|c|c|c|c|c|c|c|c|}
\hline \multirow{2}{*}{ Network form } & \multirow{2}{*}{ Supply node number } & \multicolumn{10}{|c|}{ Attack times } \\
\hline & & 0 & 1 & 2 & 3 & 4 & 5 & 6 & 7 & 8 & 9 \\
\hline \multirow{3}{*}{ a-form (pane) } & 1 & 1.0000 & 0.7700 & 0.5638 & 0.3144 & 0.1519 & 0.0725 & 0.0275 & 0.0081 & 0.0000 & 0.0000 \\
\hline & 2 & 1.0000 & 0.7613 & 0.5469 & 0.3363 & 0.1988 & 0.0981 & 0.0413 & 0.0100 & 0.0000 & 0.0000 \\
\hline & 3 & 1.0000 & 0.7744 & 0.5581 & 0.3669 & 0.2194 & 0.1238 & 0.0500 & 0.0144 & 0.0000 & 0.0000 \\
\hline \multirow{3}{*}{ b-form (radial with loop) } & 1 & 1.0000 & 0.7788 & 0.5988 & 0.3944 & 0.2175 & 0.1119 & 0.0475 & 0.0119 & 0.0000 & 0.0000 \\
\hline & 2 & 1.0000 & 0.7963 & 0.5575 & 0.3400 & 0.1794 & 0.0819 & 0.0313 & 0.0044 & 0.0000 & 0.0000 \\
\hline & 3 & 1.0000 & 0.7569 & 0.5813 & 0.3975 & 0.2381 & 0.1169 & 0.0431 & 0.0138 & 0.0000 & 0.0000 \\
\hline \multirow{3}{*}{ c-form (radial without loop) } & 1 & 1.0000 & 0.5644 & 0.3275 & 0.1606 & 0.0794 & 0.0300 & 0.0131 & 0.0038 & 0.0000 & 0.0000 \\
\hline & 2 & 1.0000 & 0.6575 & 0.4144 & 0.2213 & 0.1138 & 0.0469 & 0.0175 & 0.0063 & 0.0000 & 0.0000 \\
\hline & 3 & 1.0000 & 0.7219 & 0.4694 & 0.2981 & 0.1913 & 0.1006 & 0.0444 & 0.0106 & 0.0000 & 0.0000 \\
\hline
\end{tabular}

TABLE 2: Changes of emergence supply mileage $L$.

\begin{tabular}{|c|c|c|c|c|c|c|c|c|c|c|c|}
\hline \multirow{2}{*}{ Network form } & \multirow{2}{*}{ Supply node number } & \multicolumn{10}{|c|}{ Attack times } \\
\hline & & 0 & 1 & 2 & 3 & 4 & 5 & 6 & 7 & 8 & 9 \\
\hline \multirow{3}{*}{ a-form (pane) } & 1 & 6.75 & 33.03 & 56.39 & 84.21 & 102.59 & 111.57 & 116.76 & 119.03 & 120.00 & 120.00 \\
\hline & 2 & 5.63 & 33.03 & 57.62 & 81.43 & 97.07 & 108.58 & 115.15 & 118.80 & 120.00 & 120.00 \\
\hline & 3 & 4.50 & 30.62 & 55.40 & 77.65 & 94.57 & 105.62 & 114.16 & 118.28 & 120.00 & 120.00 \\
\hline \multirow{3}{*}{ b-form (radial with loop) } & 1 & 5.75 & 31.88 & 52.68 & 75.93 & 95.59 & 107.42 & 114.60 & 118.65 & 120.00 & 120.00 \\
\hline & 2 & 4.25 & 28.80 & 56.30 & 81.32 & 99.44 & 110.49 & 116.33 & 119.48 & 120.00 & 120.00 \\
\hline & 3 & 3.00 & 32.04 & 52.47 & 74.20 & 92.38 & 106.31 & 114.91 & 118.35 & 120.00 & 120.00 \\
\hline \multirow{3}{*}{ c-form (radial without loop) } & 1 & 3.00 & 53.92 & 81.56 & 101.07 & 110.59 & 116.43 & 118.43 & 119.55 & 120.00 & 120.00 \\
\hline & 2 & 2.13 & 42.56 & 71.03 & 93.81 & 106.54 & 114.42 & 117.90 & 119.25 & 120.00 & 120.00 \\
\hline & 3 & 1.50 & 34.27 & 64.42 & 84.74 & 97.32 & 107.98 & 114.68 & 118.73 & 120.00 & 120.00 \\
\hline
\end{tabular}

supply mileage emulation results when attack time is 0 .

(2) Effective demand ratio of c-form (radial without loop) network falls the fastest (the average value of $\mathrm{c}$-form (radial without loop) network decreases by $35.21 \%$, $37.98 \%$, and $44.68 \%$ during the first three attacks, well above $22.27 \%, 25.43 \%$, and $34.92 \%$ of b-form (radial with loop) network and 23.15\%, 27.62\%, and 39.01\% of a-form (pane) network), and its emergence supply mileage increases the fastest, which shows that the reliability of this form of network is relatively low under attack with the same network density.

(3) Generally speaking, the reliability of both b-form (radial with loop) network and a-form (pane) network is good, with the former a little better than the later. (In a pecking order from top to bottom after three attacks the rank of effective demand node ratio is b3[0.3975], b1[0.3944], a3[0.3669], b2[0.3400], a2[0.3363], and a1[0.3144].)

(4) Network efficiency shows different changing regularities in line with the selection of different supply nodes in the same form of network. In a-form (pane) network and c-form (radial without loop) network, from fast to slow the rate of decay of effective reliability ratio $P$ is, in order, 1,2 , and 3 , and from slow to fast emergence supply mileage is, in order, 1,2 , and 3 , which shows that when demand node 3 is taken as the supply node, the network has the highest reliability, followed by demand node 2 , and demand node 1 is the lowest. In b-form (radial with loop) network, from fast to slow the rate of decay of effective reliability ratio $P$ is, in order, 2,1 , and 3 , and from slow to fast emergence supply mileage is, in order, 2, 1, and 3, which shows that when demand node 3 is taken as the supply node, the network has the highest reliability, followed by demand node 1 , and demand node 3 is the lowest.

(5) There is an inverse correlation between effective demand ratio and emergence supply mileage; the lower the effective demand nodes are, the farther the emergence supply mileage is.

Taken together, the selection of network forms and supply nodes affect their corresponding emergency logistics network reliability greatly. According to different network forms, the reliability of b-form (radial with loop) network is superior to a-form (pane) network, and that of c-form (radial without loop) network is the worst; in line with the selection supply nodes, those nodes, which have high degree and wider radiation and are near the network center, are more reliable.

\section{Conclusions and Prospects}

The topological model of emergency logistics network and its emulation methods are proposed in the essay first; then 
with three typical forms of emergency logistics network as examples, emulation analyses of the three forms of emergency logistics with same density are conducted; and finally, the effective demand node ratio and changing curve of emergence supply mileage of every form of the network under different selection of supply nodes are obtained. The result shows that emergency logistics network reliability is largely affected by network forms and the selection of supply nodes. Emergency logistics network is a hotspot research subject both home and abroad in recent years; and thus, the emulation analysis thought of this thesis can inspire a new thinking in this field, and methods issued and results obtained in this essay may provide guidance and are of enlightening significance for designing an emergency logistics network with high reliability.

It is presumed that once there are connections between demand nodes and supply nodes the task of emergence material supply can be fulfilled, without considering the volume of emergence material demand and actual available supply, which simplifies actual situations. Meanwhile, the attacks of network nodes are considered, but not the attacks of edges which may bring local failure or paralysis to the network. Therefore, the emulation results will be closer to actual situations if actual freight volume and attacks of edges are considered in reliability emulation analysis of emergency logistics network, which is the emphasis of further studies.

\section{Acknowledgments}

The paper is funded by the National Natural Science Foundation of China (project no. 51009060), the Fundamental Research Funds for the Central Universities (project no. 2011B10114), Science and Technology Projects Plan of the Ministry of Housing and Urban-Rural Development of PRC (no. 2010-R2-6), and the Priority Academic Program Development of Jiangsu Higher Education Institutions (Coastal Development Conservancy).

\section{References}

[1] Z. W. Ou, H. Y. Wang, D. L. Jiang et al., "Emergency logistics," Journal of Chongqing University, vol. 27, no. 3, pp. 164-167, 2004.

[2] S. Tufekci and W. A. Wallace, "The emerging area of emergency management and engineering," IEEE Transactions on Engineering Management, vol. 45, no. 2, pp. 103-105, 1998.

[3] F. T. Boesch and R. E. Thomas, "On graphs of invulnerable communication nets," IEEE Transactions on Circuit Theory, vol. 17, no. 2, pp. 183-192, 1970.

[4] W. Yeh, "Multistate network reliability evaluation under the maintenance cost constraint," International Journal of Production Economics, vol. 88, no. 1, pp. 73-83, 2004.

[5] G. Levitin, "Reliability evaluation for acyclic consecutively connected networks with multistate elements," Reliability Engineering and System Safety, vol. 73, no. 2, pp. 137-143, 2001.

[6] T. Aven, "Reliability evaluation of multistate systems with multistate components," IEEE Transactions on Reliability, vol. 34, no. 5, pp. 473-479, 1985.

[7] C. Jane, J. Lin, and J. Yuan, "Reliability evaluation of a limitedflow network in terms of minimal cutsets," IEEE Transactions on Reliability, vol. 42, no. 3, pp. 354-361, 1993.
[8] Y.-K. Lin, "A simple algorithm for reliability evaluation of a stochastic-flow network with node failure," Computers \& Operations Research, vol. 28, no. 13, pp. 1277-1285, 2001.

[9] Y. Lin, "Using minimal cuts to evaluate the system reliability of a stochastic-flow network with failures at nodes and arcs," Reliability Engineering and System Safety, vol. 75, no. 1, pp. 4146, 2002.

[10] B. G. Gong, X. Chen, and C. Z. Hu, "Fuzzy entropy clustering approach to evaluate the reliability of emergency logistics system," Energy Procedia A, vol. 16, pp. 278-283, 2012.

[11] G. Levitin and A. Lisnianski, "A new approach to solving problems of multi-state system reliability optimization," Quality and Reliability Engineering International, vol. 17, no. 2, pp. 93104, 2001.

[12] C. Hsieh and Y. Chen, "Resource allocation decisions under various demands and cost requirements in an unreliable flow network," Computers and Operations Research, vol. 32, no. 11, pp. 2771-2784, 2005.

[13] C. Hsieh and M. Lin, "Reliability-oriented multi-resource allocation in a stochastic-flow network," Reliability Engineering and System Safety, vol. 81, no. 2, pp. 155-161, 2003.

[14] A. M. Caunhye, X. Nie, and S. Pokharel, "Optimization models in emergency logistics: a literature review," Socio-Economic Planning Sciences, vol. 46, no. 1, pp. 4-13, 2012.

[15] W. Xu, S. He, R. Song, and J. Li, "Reliability based assignment in stochastic-flow freight network," Applied Mathematics and Computation, vol. 211, no. 1, pp. 85-94, 2009.

[16] M. Chang, Y. Tseng, and J. Chen, "A scenario planning approach for the flood emergency logistics preparation problem under uncertainty," Transportation Research E, vol. 43, no. 6, pp. 737754, 2007.

[17] Y. Lin and C. Yeh, "Optimal carrier selection based on network reliability criterion for stochastic logistics networks," International Journal of Production Economics, vol. 128, no. 2, pp. 510517, 2010.

[18] T. Jin and Y. Tian, "Optimizing reliability and service parts logistics for a time-varying installed base," European Journal of Operational Research, vol. 218, no. 1, pp. 152-162, 2012.

[19] B. Vahdani, R. Tavakkoli-Moghaddam, and F. Jolai, "Reliable design of a logistics network under uncertainty: a fuzzy possibilistic-queuing model," Applied Mathematical Modelling, vol. 37, no. 5, pp. 3254-3268, 2013.

[20] J. Sheu, "Dynamic relief-demand management for emergency logistics operations under large-scale disasters," Transportation Research E, vol. 46, no. 1, pp. 1-17, 2010.

[21] P. Peng, L. V. Snyder, A. Lim, and Z. Liu, "Reliable logistics networks design with facility disruptions," Transportation Research $B$, vol. 45, no. 8, pp. 1190-1211, 2011.

[22] G. J. Ji and C. H. Zhu, "A study on emergency supply chain and risk based on urgent relief service in disasters," Systems Engineering Procedia, vol. 5, pp. 313-325, 2012.

[23] S. M. Hong-Minh, S. M. Disney, and M. M. Naim, "The dynamics of emergency transshipment supply chains," International Journal of Physical Distribution \& Logistics Management, vol. 30, no. 9, pp. 788-817, 2000.

[24] Y. Lin, "A stochastic model to study the system capacity for supply chains in terms of minimal cuts," International Journal of Production Economics, vol. 124, no. 1, pp. 181-187, 2010.

[25] M. U. Thomas, "Supply chain reliability for contingency operations," in Proceedings of the Annual Reliability and Maintainability Symposium, pp. 61-67, Seattle, Wash, USA, January 2002. 
[26] B. Dengiz, F. Altiparmak, and A. E. Smith, "Efficient optimization of all-terminal reliable networks, using an evolutionary approach," IEEE Transactions on Reliability, vol. 41, no. 1, pp. 1826, 1997.

[27] S. Peyer, D. Rautenbach, and J. Vygen, "A generalization of Dijkstra's shortest path algorithm with applications to VLSI routing," Journal of Discrete Algorithms, vol. 7, no. 4, pp. 377390, 2009. 


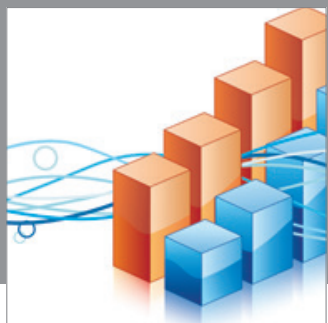

Advances in

Operations Research

mansans

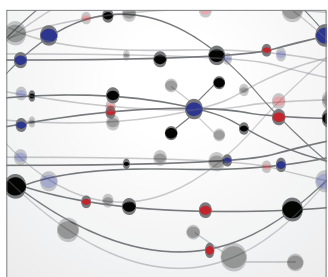

The Scientific World Journal
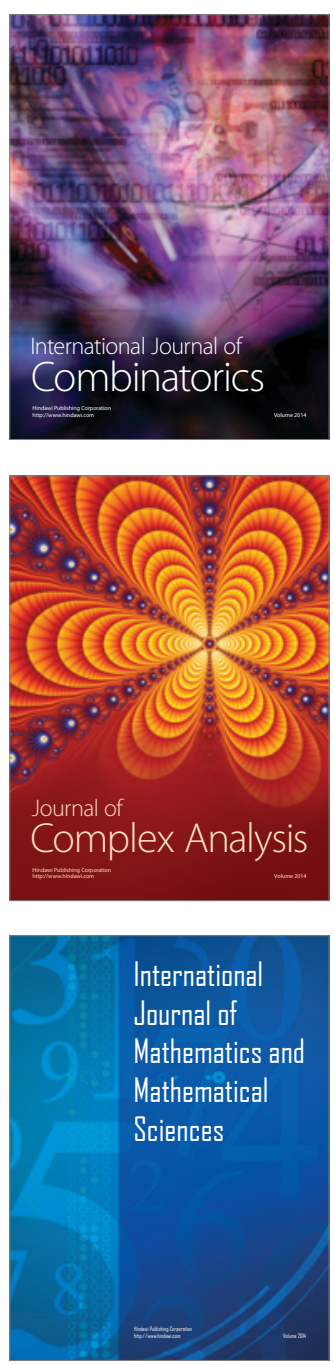
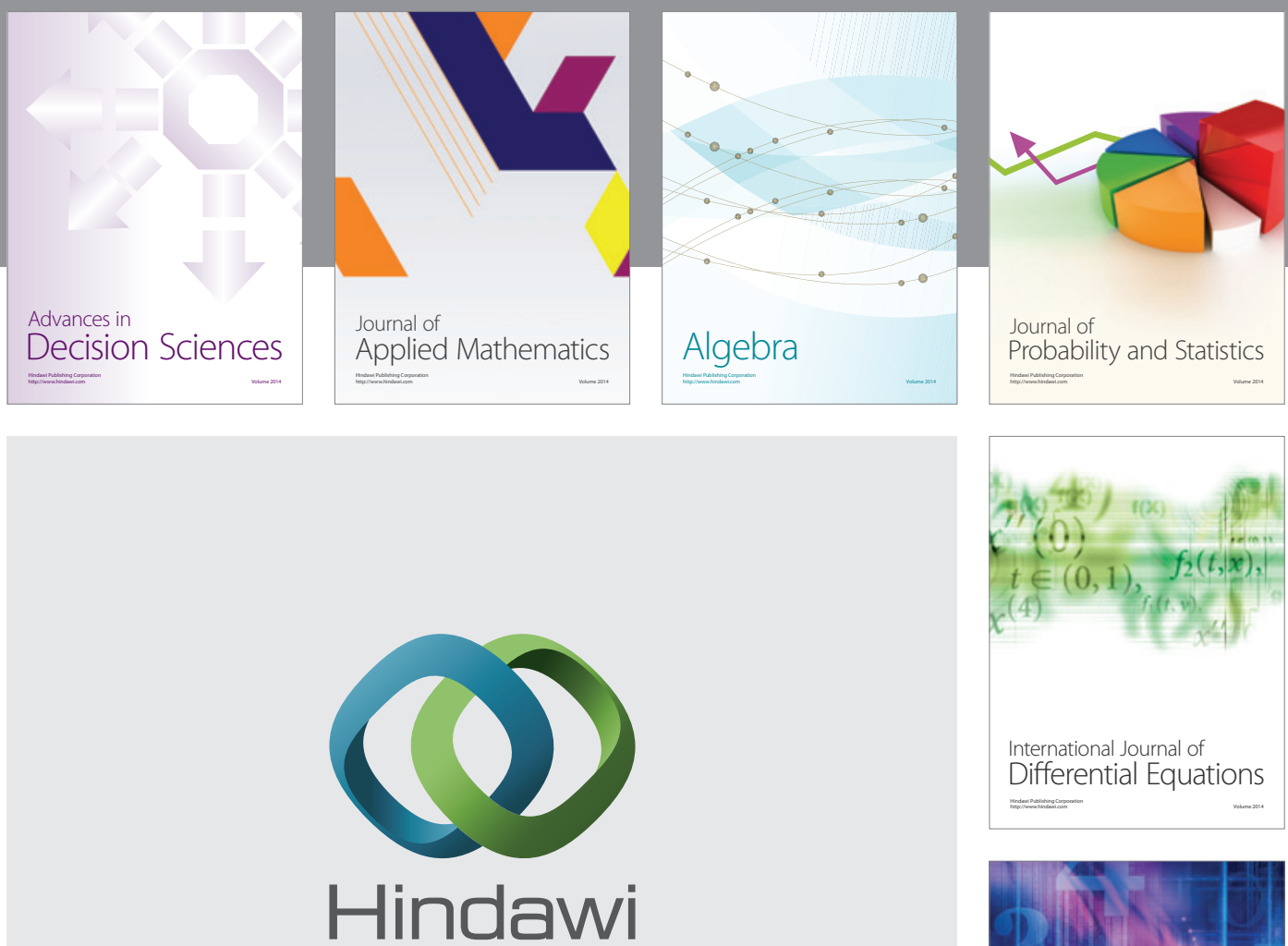

Submit your manuscripts at http://www.hindawi.com
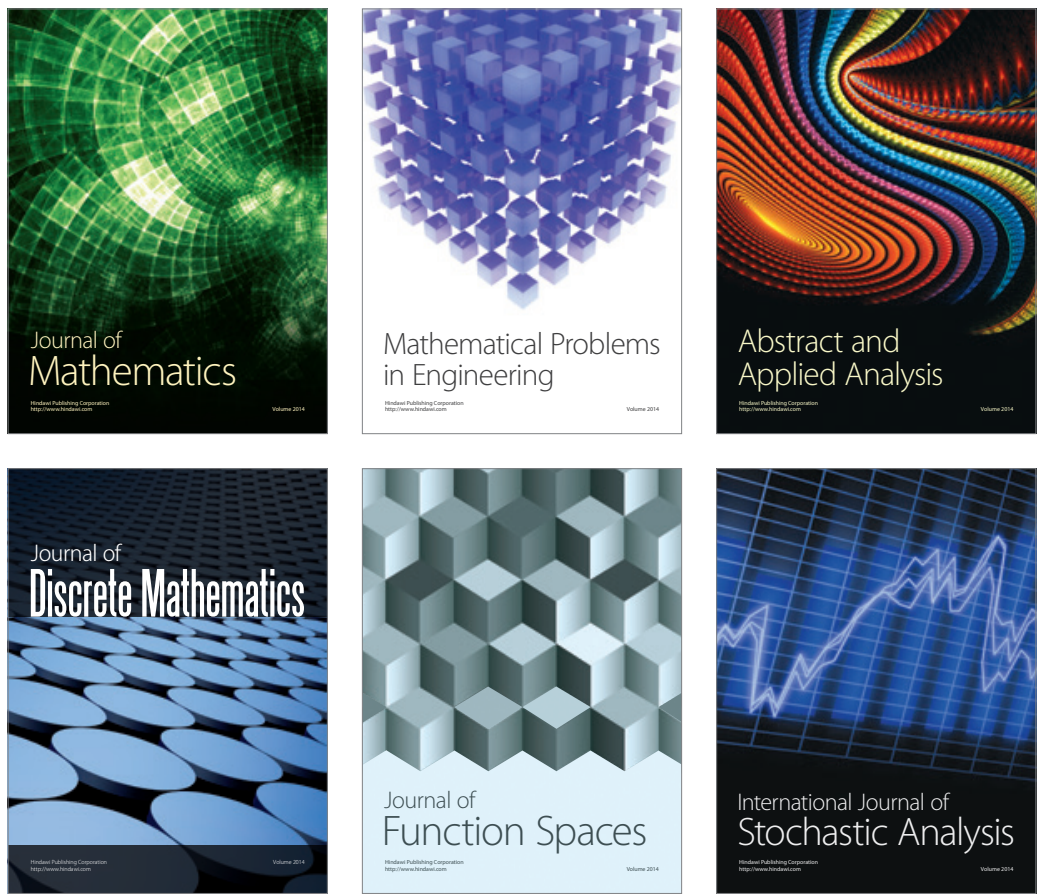

Journal of

Function Spaces

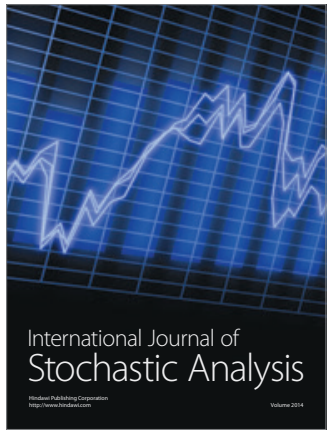

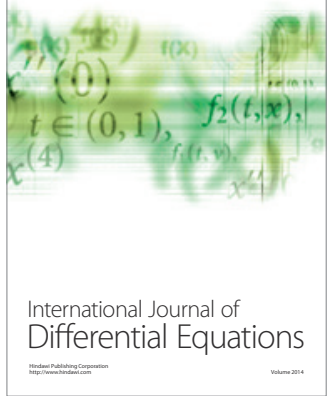
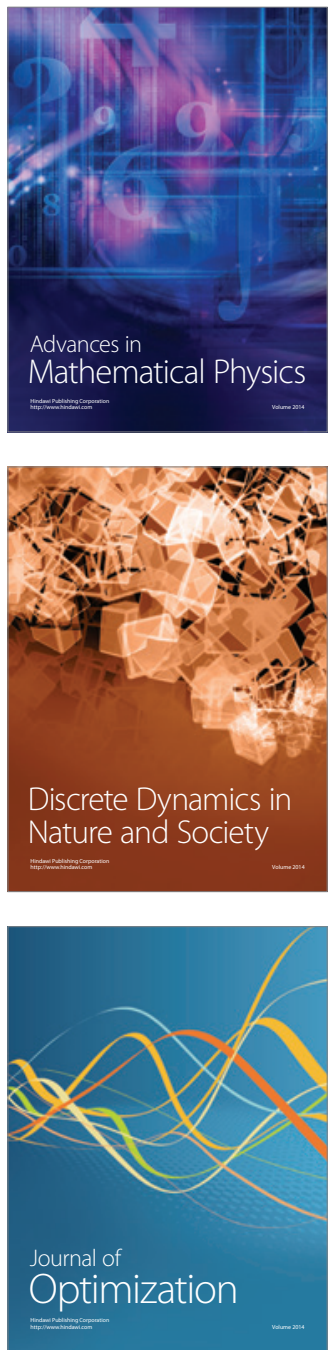